\title{
PILOT INJECTOR REDESIGN TO REDUCE N+3 CYCLE EMISSIONS FOR A GAS-TURBINE COMBUSTOR
}

\author{
Kumud Ajmani \\ Vantage Partners, LLC \\ Cleveland, Ohio, USA \\ Clarence Chang \\ NASA Glenn Research Center \\ Cleveland, Ohio, USA
}

\author{
Phil Lee \\ Woodward FST, Inc \\ Zeeland, MI, USA \\ Kathleen M. Tacina \\ NASA Glenn Research Center \\ Cleveland, Ohio, USA
}

An overview is given of an effort for the use of CFD analysis to complement design and configuration definition of third generation Lean-Direct Injection combustion concepts (LDI-3) for NASA's N+3 program. The National Combustion Code (OpenNCC) was used to perform non-reacting and two-phase reacting flow computations for a three-cup, nineteen-element flametube array with redesigned pilot injectors to improve spray and emissions characteristics when compared to a previous LDI-3 design. All computations were performed with a consistent approach to mesh-generation, spray modeling, ignition and kinetics modeling for a 'medium-power' cycle condition. Computational predictions of the aerodynamics of a new pre-filming pilot injector were used to arrive at an optimized aerothermal design that meets effective area and fuel-air mixing criteria. The newly designed pilot injectors were shown to provide considerable improvements in aerodynamic stability, flame-tube pattern factor and NOx emissions, when compared to the original design.

\section{INTRODUCTION}

NASA's third-generation $(\mathrm{N}+3)$ aeronautics efforts under the Advanced Air Vehicles program set more stringent emissions and performance goals for gas-turbine systems for single-aisle, mid-range aircraft of $25000 \mathrm{lb}$. thrust class at an operating takeoff pressure ratio of up to 40 [1]. Some details of a thirdgeneration multi-point Lean-Direct injection configuration (LDI-3) to meet NASA's N+3 goals were described in [2]. The three-cup flame-tube design proposed by Woodward FST Inc consisted of three multi-element modules (or cups), and leveraged several lessons learned from extensive experimental testing [3]' and CFD evaluation [4]' of NASA's previous generation N+2 combustor designs [5]'.

The National Combustor Code (OpenNCC) is a state-of-the-art computational tool that is capable of solving the time-dependent, Navier-Stokes equations with chemical reactions. The software has been developed primarily at NASA GRC in order to support combustion simulations for a wide range of applications and has been extensively validated and tested for low-speed chemically reacting flows. Several 'best practices' for the use of OpenNCC for LDI injector design were achieved by extensive 'anchoring' [6] with available LDI-1 data [7]. The LDI-1 anchoring work enabled the use of OpenNCC in the evaluation of LDI-2 designs [4] (NASA N+2 program) and advanced CFD screening of LDI-3 designs [8] (NASA N+3 program) at NASA GRC.

The primary goal of the efforts reported in this paper was to use OpenNCC to evaluate a proposed redesign of the three-cup LDI-3 flametube array designed by Woodward, FST Inc. (WFST) for NASA's $\mathrm{N}+3$ program. The current re-design effort follows the recommendations from the CFD analysis performed from previous LDI-3 injector and flametube designs [2,9]. Geometry parameters studied with OpenNCC in the current effort included changing the turning angle, air-flow splits, and the orientation (counter or corotating) of the proposed pre-filming pilot injector for each of the three-cups of the original, 'baseline' nineteen-element flametube design. The goal was to arrive at an updated configuration that would meet the design requirements of maximum effective area, 'optimal' size of primary recirculation zone and emissions improvements when compared to the original, pressure-atomizer fueled pilot injectors [10, 11]. 
Figure 1 shows a representation of the newly designed pre-filming injector, as tested in a 19-element flametube configuration at NASA GRC. A schematic of the computational domain used for the CFD evaluation with OpenNCC, and a portion of the surface mesh for the nineteen injectors of the three-cup configuration, are shown in figure 2 .
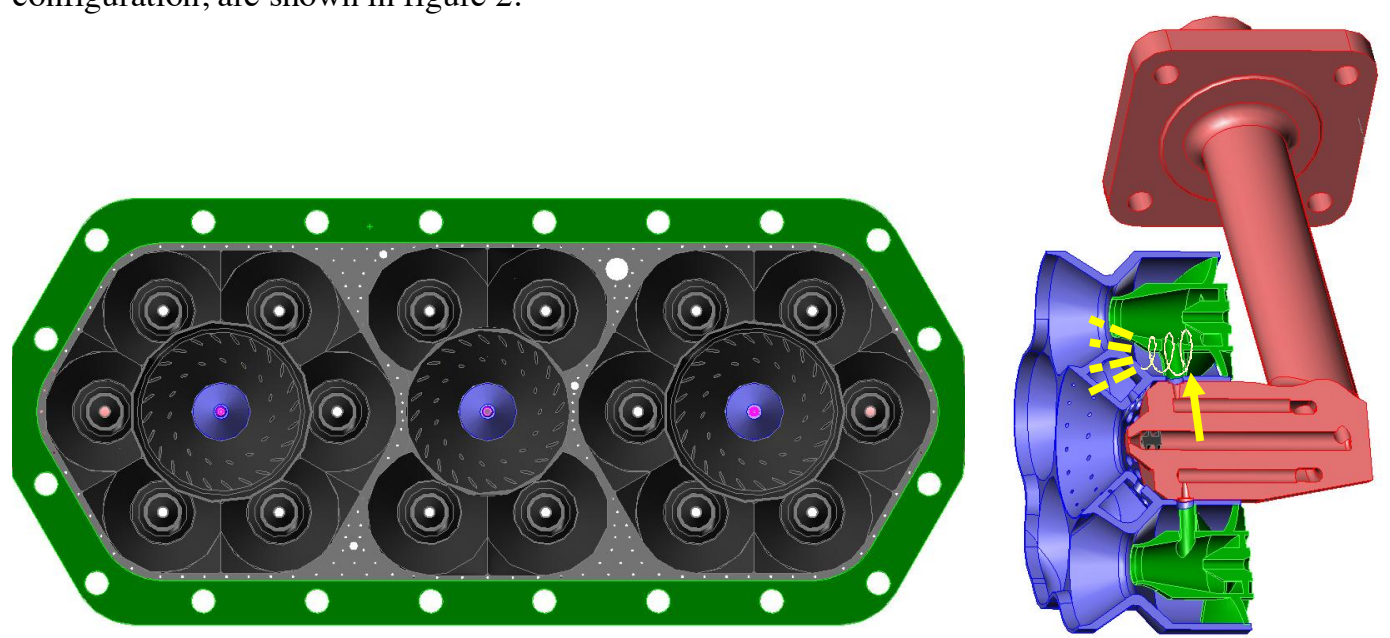

Figure 1. Dome-layout with alternating seven- and five-injector modules for an LDI-3 injector array (left) and details of the pre-filming injector (right)
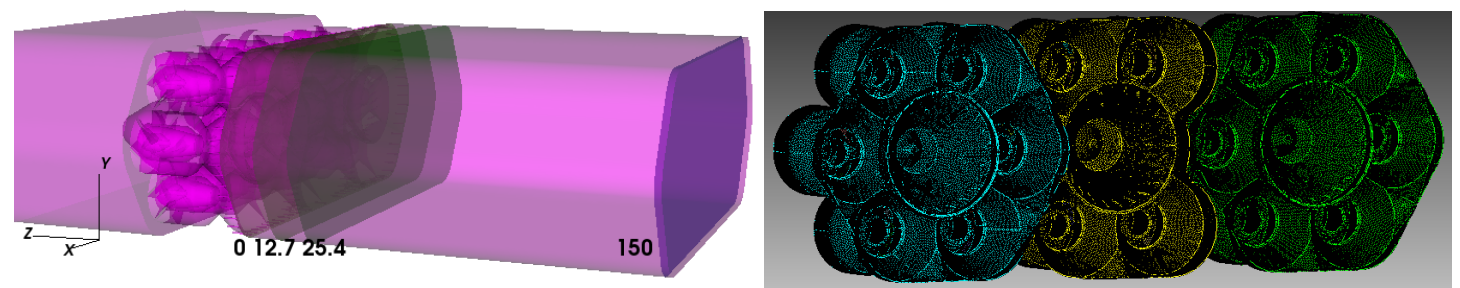

Figure 2. Left: Computational Domain for OpenNCC CFD (Dump plane: 0mm; Exit plane: 150mm). Right: Surface mesh for pilot and main injector elements of 3-cup configuration.

\section{NINETEEN ELEMENT FLAMETUBE - PILOT INJECTOR REDESIGN}

A comparison of OpenNCC CFD emissions predictions for a 'baseline', three-cup, nineteen injector element LDI-3 flametube array was reported in [5]. The comparison between OpenNCC CFD and experimental data [6] focused on two low-power cycle conditions (7\% and $30 \%$ power). In order to improve the emissions produced by the 'baseline' (also designated ' $v 3$ ') flametube, a new design (designated ' $\mathrm{v} 4$ ') was proposed, based on insights provided by OpenNCC CFD. The major changes between the ' $\mathrm{v} 3$ ' and ' $\mathrm{v} 4$ ' design were:

- Use partially pre-filming pilot injectors (v4) instead of non pre-filming Pilot injectors (v3). The ' $\mathrm{v} 4$ ' pilot injectors split the airflow into an inner and an outer stream, and the fuel spray is injected onto a short pre-filmer. These two changes are proposed to provide better fuel vaporization and mixing of fuel with the two streams of counter-rotating airflow, and hence reduce emissions and increase pilot operability and flame stability.

- All airflow passages in the Main injection elements provide co-rotating airflow (v4), instead of the alternating counter-rotating airflow between adjacent Main elements (v3) in each cup

- Cooling flow are in the pre-filming pilot injector venturi (v4) was increased by $35 \%$ as compared to the baseline pilot (v3). Cooling flow area at the dome was increased by $10 \%$. 


\section{NON-REACTING FLOW CFD COMPUTATIONS}

\section{OpenNCC Effective Area Computations}

The first criteria to be met when components of an existing injector are redesigned is to ensure that the effective area $\left(\mathrm{AC}_{\mathrm{d}}\right)$ of the new design (v4) is within $1 \%$ to $2 \%$ of the baseline (v3) design. The new design was evaluated with OpenNCC (non-reacting flow) to compute the effective area of the individual components (main injectors, pilot injectors, cooling flow holes). A typical computation proceeded as follows:

- Boundary Conditions: Fix $\mathrm{P}_{\mathrm{t}}$ (based on $\mathrm{P}_{3}=8.96 \mathrm{kPa}$ ) and $\mathrm{T}_{\mathrm{t}}$ (based on $\mathrm{T}_{3}=811 \mathrm{~K}$ ) at each inflow boundary (pilot, mains, cooling flow); Fix pressure $\left(\mathrm{P}=\mathrm{P}_{3}-\Delta \mathrm{p}\right)$ at outflow corresponding to $\Delta \mathrm{p}=3 \%$

- Run OpenNCC RANS at CFL $=0.75$ until $<0.1 \%$ mass-flow imbalance convergence is achieved (typically 100,000 steps). Use converged RANS solution to run and additional 10,000 time-steps $(\Delta \mathrm{t}=1 \mathrm{e}-6 \mathrm{~s})$ with OpenNCC TFNS (Time-Filtered Navier-Stokes).

- Compute $\mathrm{AC}_{\mathrm{d}}$ from OpenNCC TFNS prediction of mass flow rate for each inflow boundary.

A comparison of the effective area for the new pre-filming pilot design (v4), the baseline design (v3) and the experimentally measured values for the baseline design are shown in Table 1 . The new design maintains the $\mathrm{AC}_{\mathrm{d}}$ values in the primary components (main and pilot injectors), as the predicted $\mathrm{AC}_{\mathrm{d}}$ values for the new design are within 1-2\% of the baseline (v3). In addition, the intended cooling flow increases in the pilot venturi $(+35 \%)$ and the dome $(+10 \%)$ for the new design (v4) are captured very well by the OpenNCC CFD results.

\begin{tabular}{|l|c|c|c|c|}
\hline \multicolumn{1}{|c|}{ Components } & $\begin{array}{c}\text { Computed } \\
\mathrm{AC}_{\mathrm{d}}\left(\mathrm{in}^{2}\right)(\mathbf{v 4})\end{array}$ & $\begin{array}{c}\text { Computed } \mathrm{AC}_{\mathrm{d}} \\
\left(\mathrm{in}^{2}\right)(\mathbf{v} \mathbf{3})\end{array}$ & $\begin{array}{c}\text { Measured } \mathrm{AC}_{\mathrm{d}} \\
\left(\mathrm{in}^{2}\right)(\mathbf{v} \mathbf{3})\end{array}$ & $\begin{array}{c}\% \mathrm{AC}_{\mathrm{d}} \text { change } \\
(\mathbf{v 4}-\mathbf{v 3}) / \mathbf{v 3}\end{array}$ \\
\hline Main Injectors (16) & 2.413 & 2.4323 & $2.3613^{*}$ & $-0.8 \%$ \\
\hline Pilot Injectors (3) & 0.327 & 0.3348 & 0.3104 & $-2.3 \%$ \\
\hline $\begin{array}{l}\text { Pilot Cooling Holes (2 and 4 } \\
\text { rows of holes per pilot for v3 } \\
\text { and v4, respectively) }\end{array}$ & 0.117 & 0.0433 & $\begin{array}{c}\text { *(included in } \\
\text { Mains) }\end{array}$ & $35 \%$ \\
\hline Dome Face Cooling Holes & 0.0456 & 0.0418 & $\begin{array}{c}\text { *(included in } \\
\text { Mains) }\end{array}$ & $9.1 \%$ \\
\hline Total & $\mathbf{2 . 9 0 2 6}$ & $\mathbf{2 . 8 5 2 2}$ & $\mathbf{2 . 6 7 1 7}$ & $\mathbf{1 . 8 \%}$ \\
\hline
\end{tabular}

$\left(A C_{d}\right)_{C F D}=\frac{\dot{m}_{C F D}}{\sqrt{2 \times \rho \times \triangle P \text { data }}}$; here $\Delta P_{\text {data }}=26882 \mathrm{~Pa}$ was used for calculating $\mathrm{AC}_{\mathrm{d}}$.

Table 1. Effective area $\left(\mathrm{AC}_{\mathrm{d}}\right)$ values computed by OpenNCC for pre-filming (v4) vs baseline (v3) pilot flametube configuration

\section{OpenNCC Non-Reacting Flow Comparisons}

Figures 3 and 4 show axial-velocity contours of the OpenNCC results at various cross-sections through the two configurations of the, three-cup, nineteen-element flametube array with pre-filming pilot (v4) and 'baseline' pilot injectors (v3). Contours for both configurations are shown for non-reacting, time-averaged solutions obtained from a time-accurate, time-filtered Navier-Stokes (TFNS) approach [12]. Both the solutions are obtained using similar meshes, comprising of approximately 15 million tetrahedral mesh elements. 
A strong, prominent central recirculation zone (CRZ) behind each pilot is predicted by OpenNCC for both configurations. Note that each of the top and bottom plots are for the pilot element that is at the center of each of the seven-element cups, while the middle plot is for the slightly recessed pilot element of the five-element cup. OpenNCC TFNS does predict a slightly stronger, more symmetric, and larger recirculation zone behind each of the pre-filming pilots (v4), as compared to the 'baseline' pilots (v3). In addition, the 'baseline' pilot shows no corner recirculation zone (CoRZ) on the walls of the pilot venturis. The stronger, more uniform CRZ and the absence of any CoRZ for the redesigned pilot are both deemed to be desirable characteristics for the stability and performance of the pilot injectors.
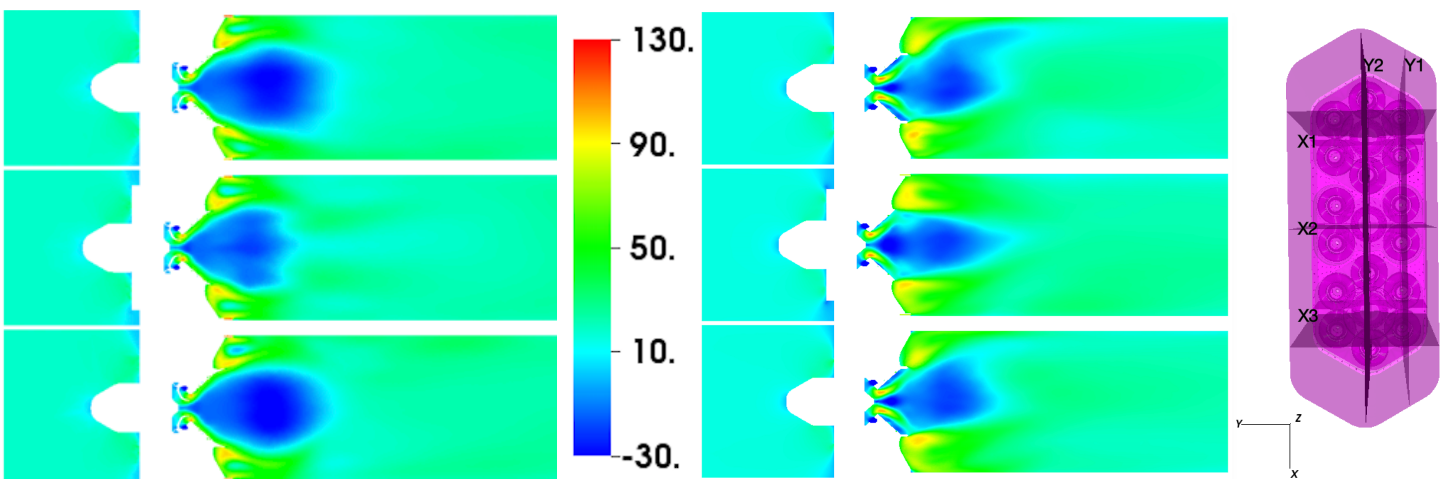

Figure 3. Axial velocity $(\mathrm{m} / \mathrm{s})$ contours in three axial cross-sections $(\mathrm{X} 1, \mathrm{X} 2, \mathrm{X} 3)$ through the centerlines of the three pilot injectors. Left: Pre-filming Pilot (v4). Right: Baseline Pilot (v3)

Figure 4 shows a cross-section through the central plane of the three-cup flametube (three pilot injectors, each with two neighboring main injectors). The CRZ behind the pilot injectors is stronger, and better defined for the redesigned pilot elements. There is a significantly smaller CRZ behind the pilot injector of the central cup of the new configuration, as compared to the baseline configuration. This reduction in recirculating mass would reduce the residence time, and hence emissions produced by the new pre-filming pilot injectors.
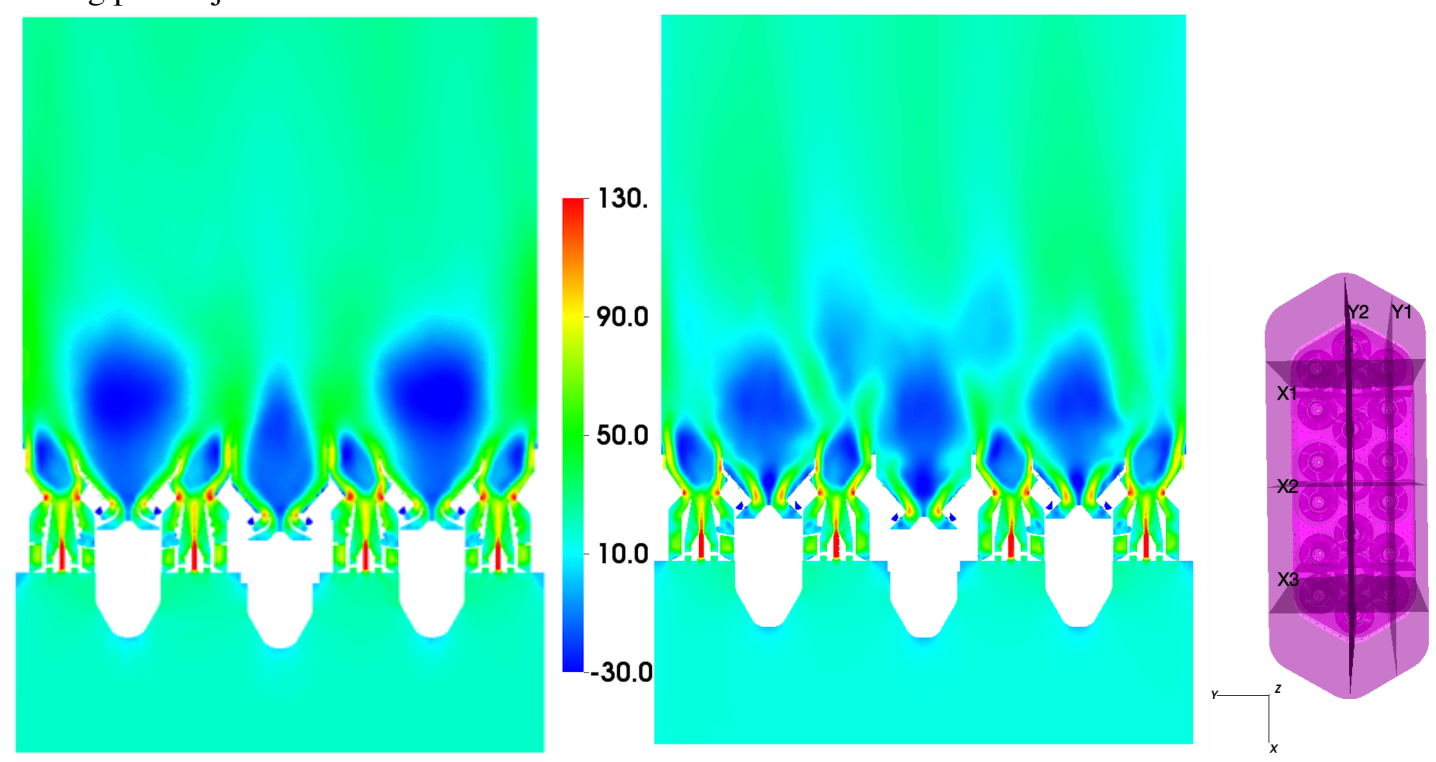

Figure 4. Axial velocity $(\mathrm{m} / \mathrm{s})$ contours at the centerline cross-section of the flame-tube assembly (Y2). Left: Pre-filming Pilot (v4). Right: Baseline Pilot (v3) 
Figure 5 shows a comparison of axial velocity contours at the dump plane of both the three-cup flametubes. The CRZ behind the pilot injectors is stronger, and better defined for the redesigned pilot elements. As expected, the recirculation zones behind all of the main injectors for the two configurations are fairly similar, as there is no change in the swirler angles between v3 and v4. The impact of changing the swirl orientation of adjacent main injectors so that all of them have co-rotating swirlers, can be seen in the overall smoother aerodynamics of the redesigned three-cup flametube (v4).

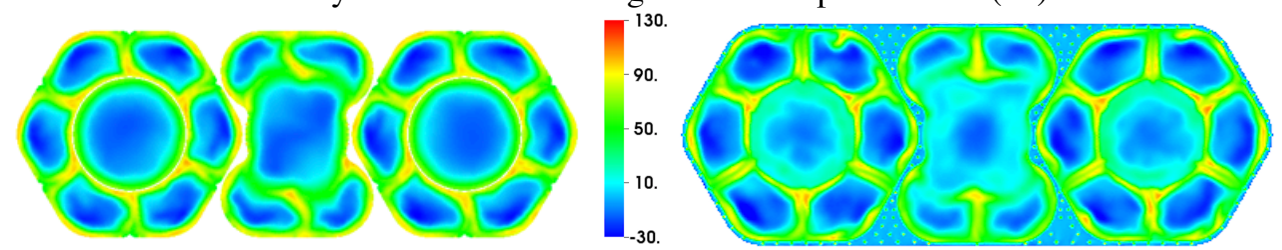

Figure 5. Axial velocity $(\mathrm{m} / \mathrm{s})$ contours at the dump-plane cross-section of the flame-tube assembly. Left: Pre-filming Pilot (v4). Right: Baseline Pilot (v3)

\section{OpenNCC Reacting Flow Comparisons - Pre-filming Pilots (v4) and Baseline Pilots (v3)}

A comparison of the reacting flowfield behind the 'baseline' non-pre-filming pilot configuration (v3) and the redesigned pre-filming pilot configuration (v4) is described in this section. The non-reacting OpenNCC TFNS solution is used as the initial condition for the reacting flow computations. The computed conditions represent a 'medium-power' $\mathrm{N}+3$ ICAO cycle condition [10] of $\mathrm{P}_{3}=8.96 \mathrm{MPa}, \mathrm{T}_{3}=811 \mathrm{~K}, \Delta \mathrm{p}=3 \%$ and overall $\mathrm{FAR}=0.03$. The Jet-A fuel was modeled with a lagrangian spray formulation [13], and the fuel-air mixture was ignited with an ignition source term. The Jet-A/Air chemistry was modeled with a 14species, 18-step, reduced finite-rate kinetics model [14].

Figures 6 and 7 show reacting-flow axial-velocity comparisons for the two computed configurations. The results represent the time-averaged solution for the final flow through-cycle of the OpenNCC TFNS computations $(2.6 \mathrm{~m}-\mathrm{s})$. The pre-filming pilot configuration shows considerably larger CRZ behind the two outer pilots (seven-element cups), as compared to the central pilot (five-element cup). In contrast, all of the pilot CRZ for the baseline configuration are similar to each other, and are of much shorter length and strength as compared to the pre-filming pilot design. The net effect these changes in the flow patterns is that the total mass of recirculating flow in the new design (with pre-filming pilot injectors) is about $20 \%$ higher than that of the baseline configuration.
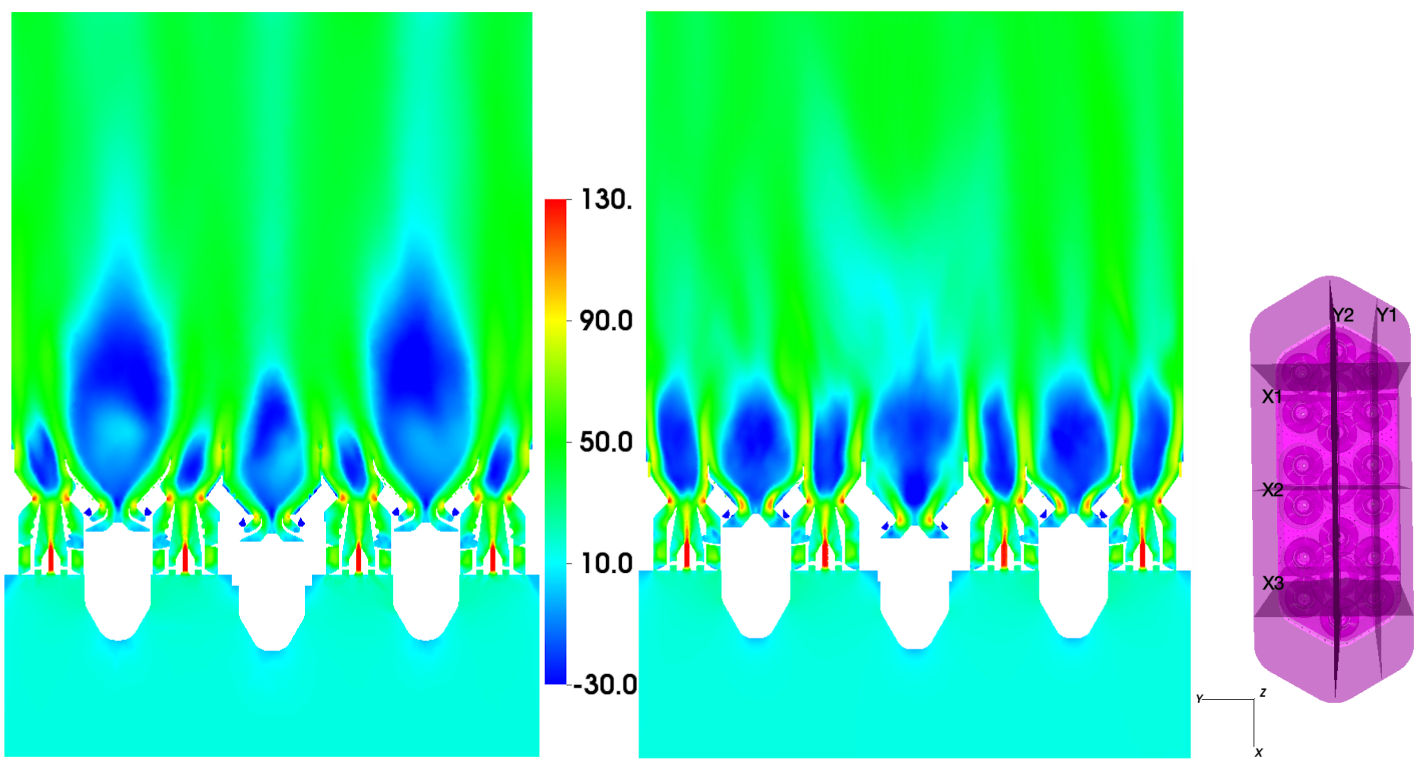

Figure 6. Axial velocity (m/s) contours (reacting flow) at the centerline cross-section of the flame-tube assembly (Y2). Left: Pre-filming Pilot (v4). Right: Baseline Pilot (v3) 

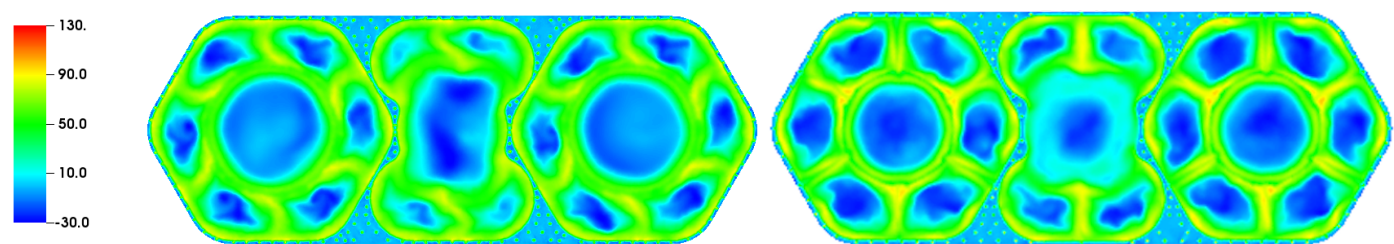

Figure 7. Axial velocity $(\mathrm{m} / \mathrm{s})$ contours (reacting flow) at the dump-plane cross-section of the 3-cup configurations. Left: Pre-filming Pilot (v4). Right: Baseline Pilot (v3)

The time-averaged temperature contours for the final flow through-cycle of the OpenNCC TFNS computations for the two configurations at two different cross-sections are shown in figures 8 and 9 . The pre-filming pilot configuration shows fairly uniform flame structure behind all three pilot injectors. In addition, the flame zones behind the pilots are much cooler for the pre-filming configuration, and there is much better mixing of the pilot and main injector flame zones downstream of the dome. The temperature contours at the dump plane (figure 9) also highlight the major differences in the flame zones behind the pilot injectors for the two configurations. In summary, the primary design changes made in the new configuration (pilots with pre-filming fuel impingement, all main injectors with co-swirling air-flow) seem to significantly impact the near-dome flame structure and improve the mixing of individual injector streams which could translate to better pattern factor, reduced NOx emissions and improved combustion efficiency.
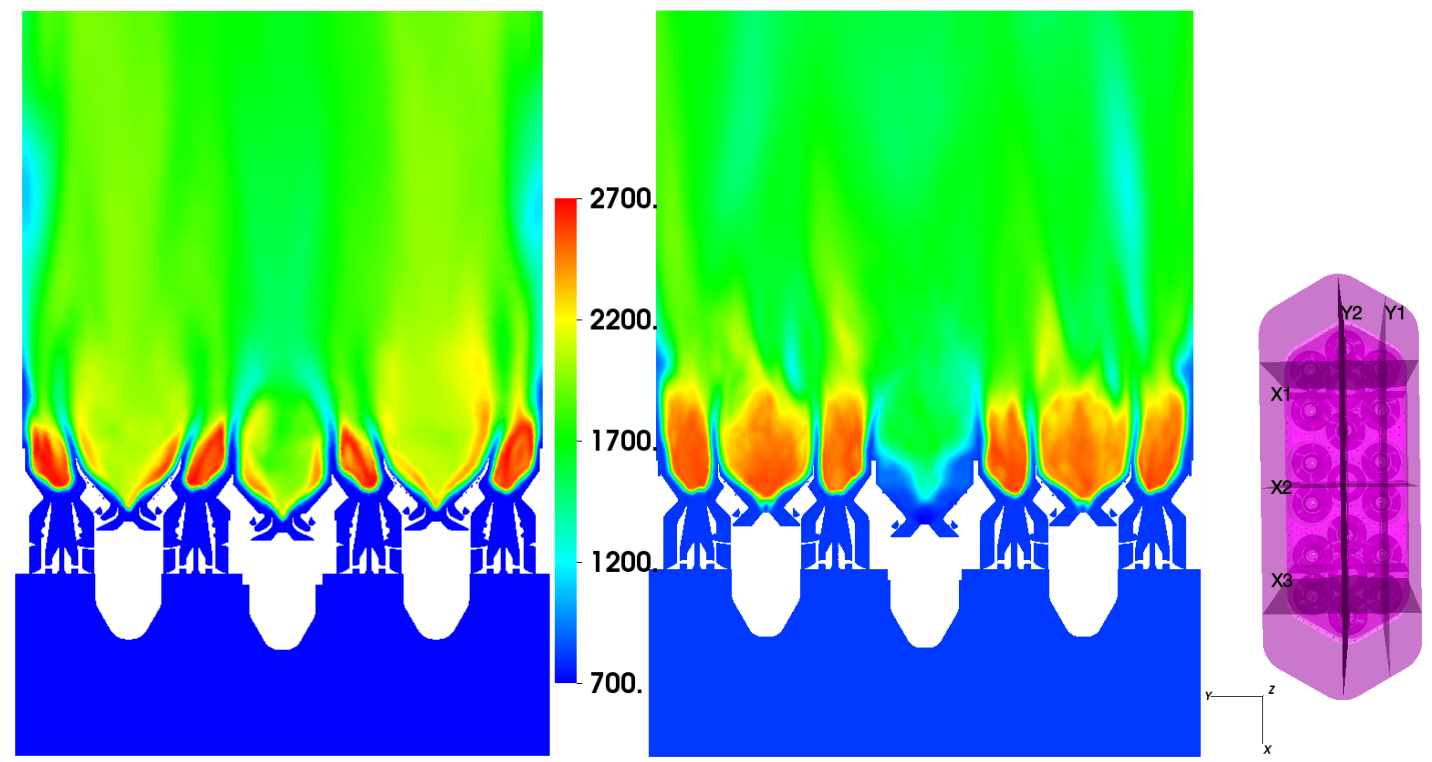

Figure 8. Temperature (K) contours (reacting flow) at the centerline cross-section of the flame-tube assembly (Y2). Left: Pre-filming Pilot (v4). Right: Baseline Pilot (v3)
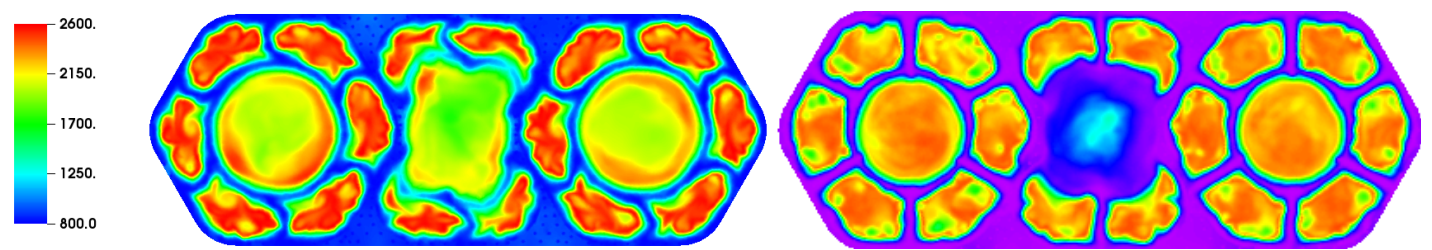

Figure 9. Temperature $(\mathrm{K})$ contours (reacting flow) at the dump-plane cross-section of the 3-cup configurations. Left: Pre-filming Pilot (v4). Right: Baseline Pilot (v3)

One of the principal objectives of this study is to determine the impact of pilot injector redesign on emissions performance of the three-cup flame-tube assembly. The time-averaged NO mass-fraction contours for the two OpenNCC TFNS configurations at two different cross-sections are shown in figures 10 
and 11. The pre-filming pilot configuration shows considerably higher NO production, particularly behind the two pilot injectors of the two outer seven-element cups. This behavior is consistent with the relatively large recirculation zones behind these two pilot injectors (see figure 6). The larger NO produced by the prefilming pilots is offset by the lower NO produced by the main injectors of the new design. The massweighted average of the EINOx ( $\mathrm{g} / \mathrm{kg}$ of fuel) computed at the exit plane of the computational domain for the 'baseline' and 'pre-filming' designs are 7.7 and 6.5, respectively. Hence, the redesigned pilot configuration with pre-filming fuel injection provides a $16 \%$ improvement in EINOx when compared with the 'baseline' design with pressure-atomizing simplex fuel nozzles.
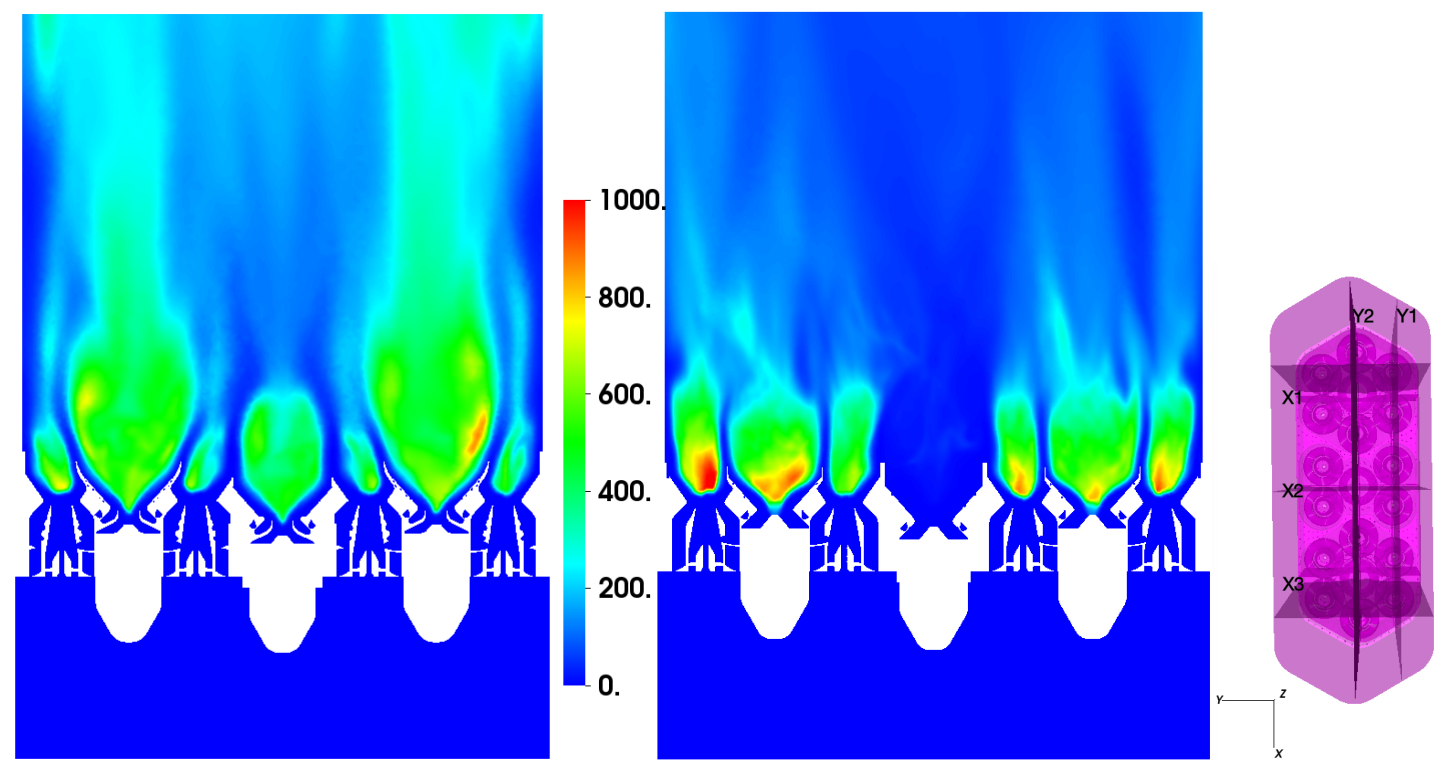

Figure 10. NO mass-fraction (*1e6) contours (reacting flow) at the centerline cross-section of the flametube assembly (Y2). Left: Pre-filming Pilot (v4). Right: Baseline Pilot (v3)
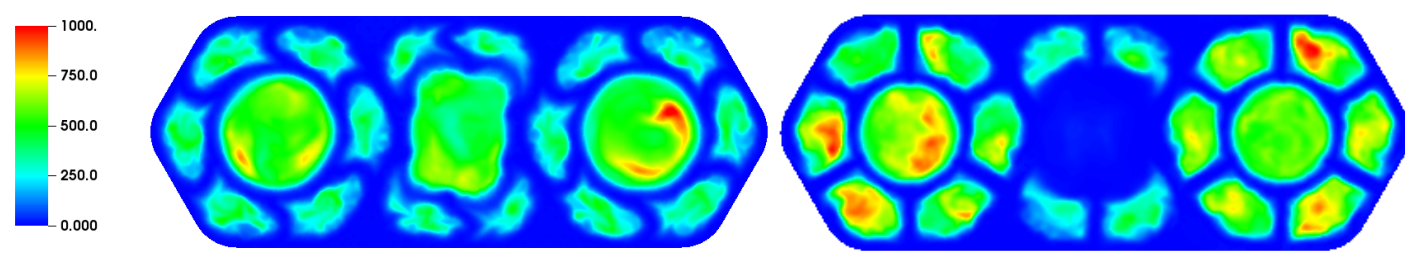

Figure 11. NO mass-fraction $(* 1 \mathrm{e} 6)$ contours (reacting flow) at the dump-plane cross-section of the 3-cup configurations. Left: Pre-filming Pilot (v4). Right: Baseline Pilot (v3)

\section{SUMMARY AND FUTURE WORK}

The National Combustor Code (OpenNCC) was used to perform CFD evaluations of two different Lean-Direct Injection (LDI) combustor flame-tube arrays designed for NASA's N+3 (LDI-3) program efforts by Woodward, FST Inc. (WFST). The three pilot injectors of the three-cup 'baseline' LDI-3 design used pressure-atomizing fuel nozzles. A new, alternative design which replaced the pressure-atomizers with fuel pre-filming nozzles was proposed and evaluated with OpenNCC CFD. Multi-phase reacting flow simulations were performed for the 'baseline' and pre-filming configurations, at conditions representative of a 'medium power' ICAO N+3 subsonic condition. The CFD results predicted significant benefits by replacing the pressure-atomizing pilots with the fuel pre-filming pilots. The new design showed better pattern factor and better EINOx emissions performance when compared to the 'baseline' design. In future work, the proposed pilot injector redesign will be further evaluated with OpenNCC to gain insight into its performance, emissions and dynamic stability at additional $\mathrm{N}+3$ cycle operating conditions (idle, approach and takeoff). The OpenNCC emissions predictions for the pre-filming pilot configuration will also be compared with experimental datasets, when they become available in the future. 


\section{ACKNOWLEDGEMENTS}

This work was funded by Advanced Air Transportation Technology (AATT) Project under NASA's Advanced Air Vehicles (AAV) Program.

\section{REFERENCES}

1. Surgenor, A., A Path to N+3 Fuel-Flexible Combustors, Green Aviation Technical Information Meeting, March 31, 2016.

2. Ajmani, K., Mongia, H.C. and Lee, P., "CFD Evaluation of a 3rd Generation LDI Combustor," AIAA Paper 2017-5017, 53rd AIAA Joint Propulsion Conference, July 2017, Atlanta GA.

3. Tacina, K. M., Podboy, D. P., He, Z. J., Lee, P., Dam, B. and Mongia, H., A Comparison of Three Second-generation Swirl-Venturi Lean Direct Injection Combustor Concepts, 52nd AIAA/SAE/ASEE Joint Propulsion Conference, AIAA Propulsion and Energy Forum, Salt Lake City UT (AIAA 2016-4891)

4. Ajmani, K., Mongia, H. C., and Lee, P., "CFD computations of emissions for LDI-2 combustors with simplex and airblast injectors", AIAA Paper 2014-3529, 50th AIAA Joint Propulsion Conference, July 2014.

5. Reddy, D. R. and Lee, C-M, An Overview of Low-Emission Combustion Research at NASA Glenn, ASME Turbo Expo 2016: Turbomachinery Technical Conference and Exposition GT2016; 13-17 Jun. 2016; Seoul S. Korea; Paper GT2016-56100

6. Ajmani, K., Mongia, H.C. and Lee, P., "CFD Best Practices to Predict NOx, CO and Lean Blowout for Combustor Design,” ASME IGTI Paper GT2013-95669, ASME Turbo Expo 2013, San Antonio TX, June 2013.

7. Tacina, R., Lee, P., and Wey, C., "A Lean-Direct-Injection Combustor Using a 9 Point Swirl-Venturi Fuel Injector," ISABE 2005-1106.

8. Ajmani, K., Mongia, H.C. and Lee, P., "CFD Based Design of a Filming Injector for N+3 Combustors," AIAA Paper 2016-4783, 52nd AIAA Joint Propulsion Conference, July 2016, Salt Lake City, UT.

9. Ajmani, K., Mongia, H.C. and Lee, P., "Parametric Design of Injectors for LDI-3 Combustors," AIAA Paper 2015-3785, 51st AIAA Joint Propulsion Conference, July 2015, Orlando, FL.

10. Tacina, K.M., Podboy, D.P., Lee, P., and Dam, B., "Gaseous Emissions Results from a Three-Cup Flametube Test of a Third-Generation Lean Direct Injection Combustor", ISABE 2017, Edinburgh UK.

11. Ajmani, K., Lee, P., Mongia, H.C. and Tacina, K.M., "CFD Predictions of N+3 Cycle Emissions for a Three-Cup Gas Turbine Combustor," AIAA Paper 2018-4957, 54th AIAA Joint Propulsion Conference, July 2018, Cincinnati OH.

12. Liu, N.-S., and Wey, C.T., "On the TFNS Subgrid Models for Liquid-Fueled Turbulent Combustion," AIAA Paper 2014-3569, AIAA Propulsion and Energy Conference, Cleveland, OH, July 2014.

13. Raju, M. S., “LSPRAY-IV: A Lagrangian Spray Module,” NASA CR-2012-217294.

14. Ajmani, K., Kundu, K., and Yungster, S., "Evaluation of Reduced Mechanisms for Combustion of JetA in LDI Combustor CFD Calculations," AIAA Paper 2014-3662, AIAA Propulsion and Energy Conference, Cleveland, OH, July 2014. 